\section{Vessel Extraction Under Non-Uniform Illumination: A Level Set Approach} K. W. Sum*, Student Member, IEEE, and
Paul Y. S. Cheung, Senior Member, IEEE

\begin{abstract}
Vessel extraction is one of the critical tasks in clinical practice. This communication presents a new approach for vessel extraction using a level-set-based active contour by defining a novel local term that takes local image contrast into account. The proposed model not only preserves the performance of the existing models on blurry images, but also overcomes their inability to handle nonuniform illumination. The efficacy of the approach is demonstrated with experiments involving both synthetic images and clinical angiograms.
\end{abstract}

Index Terms-Active contours, level set method, local contrast enhancement, medical image analysis, nonuniform illumination, vessel extraction.

\section{INTRODUCTION}

Vessel extraction is one of the critical tasks in clinical practice. The complex structure of vessels makes angiogram segmentation a challenging problem. The vasculatures are very important in planning and performing neurosurgery and cardiovascular surgery. A comprehensive review of vessel extraction techniques and algorithms can be found in [1]. In this communication, we focus on the new development of angiogram segmentation based on an active contour model.

Classical snake models [2]-[4] establish a very efficient framework for image segmentation. However, they cannot handle topological changes that are required in some applications. A level-set-based model can handle complex object boundaries with flexible curve evolutions. Since the level set function is defined in Euclidean space, it is also easily extendable to higher dimensions.

The level set method [5] was first applied to fronts propagation by Osher and Sethian [6]. It was then used in shape recovery and isolation of shapes from their background by Malladi, Sethian, and Vemuri [8]. There are a number of edge-based level-set-based models [9]-[11]. They include the geometric model defined in terms of the mean curvature motion and the geodesic model which defines the problem in Riemannian space.

Chan and Vese developed a region-based model [12], which applies global statistics to the extraction of objects. This is useful for objects with blurry boundary and homogenous intensity. In most clinical angiograms, however, the vessels are normally not homogenous in intensity. This could be due to several reasons, including limited exposure to minimize the side effects to the patient in X-ray images, the projection of the 3-D object to a 2-D image across different depths, or the artifacts of different imaging techniques. The existing active contour models using only global statistics are not capable of extracting such vessels.

Manuscript received July 20, 2006; revised March 7, 2007. Asterisk indicates corresponding author.

*K. W. Sum is with the Department of Electrical and Electronic Engineering, The University of Hong Kong, Pokfulam, Hong Kong (e-mail: kwsum@ieee. org).

P. Y. S. Cheung is with the Department of Electrical and Electronic Engineering, The University of Hong Kong, Pokfulam, Hong Kong (e-mail: cheung@eee.hku.hk).

Color versions of one or more of the figures in this paper are available online at http://ieeexplore.ieee.org.

Digital Object Identifier 10.1109/TBME.2007.896587
In this communication, we propose a new approach for vessel extraction using a region-based active contour model by incorporating a novel local term to enhance segmentation results. The efficacy of the proposed model is demonstrated with both synthetic images and clinical angiograms. The results are compared with the existing region-based method [12].

This communication is organized as follows. In Section II, the concept and the formulation of the proposed model are described. Experimental results and conclusions are given in Sections III and IV, respectively.

\section{DESCRIPTIONS OF THE MODEL}

The proposed active contour model uses a two-stage approach. The first stage extracts the vessels partially using a global term. The result is then refined in the second stage using a local term.

We define the vessel as an open subset $\omega$ of $\Omega$, where $\Omega$ is the entire image space. The evolving curve $C$ in $\Omega$ is defined as the boundary of $\omega$. The background outside $C$ denotes the region $\Omega \backslash \omega$. The overall energy function is defined as $J(C)=\alpha F_{G}(C)+\beta F_{L}(C)$, where $\alpha$ and $\beta$ are the regulating parameters. Initially, the algorithm sets $\alpha=1$ and $\beta=0$. When the global term converges, the algorithm sets $\alpha=0$ and $\beta=1$ to activate the local term for the second stage.

\section{A. Global Term}

The global term in (1) is basically adopted from the Chan-Vese model [12]. It divides the image $u_{o}$ into two statistically separated regions, which represent the vessel and the background:

$$
\begin{aligned}
& F_{G}(C)=\mu \text { Length }(C)+\lambda_{1} \int_{\omega}\left|u_{o}(x, y)-c_{1}\right|^{2} d x d y \\
& \qquad+\lambda_{2} \int_{\Omega \backslash \omega}\left|u_{o}(x, y)-c_{2}\right|^{2} d x d y
\end{aligned}
$$

where $\mu, \lambda_{1}$, and $\lambda_{2}$ are the weighting factors; Length $(C)$ is the length of the curve $C . c_{1}$ and $c_{2}$ are the average intensities of the areas inside and outside $C$, respectively.

\section{B. Local Term}

Only part of the vessels can be extracted by the global term, since some vessels are statistically different from the globally extracted portions due to nonuniform illumination. We define the novel local term [7] to address this problem in (2).

$$
\begin{aligned}
F_{L}(C)=\lambda_{3} \int_{\sigma} \mid u_{N}(x, y)- & \left.m_{1}\right|^{2} d x d y \\
& +\lambda_{4} \int_{\Phi \backslash \sigma}\left|u_{N}(x, y)-m_{2}\right|^{2} d x d y
\end{aligned}
$$

where $\lambda_{3}$ and $\lambda_{4}$ are the weighting factors, $u_{N}$ is the local contrast enhanced image generated by a sliding neighborhood function as in (3), and $m_{1}$ and $m_{2}$ are the average values of $u_{N}$ inside and outside $C$, respectively. The local term is applied only in the narrowband $\Phi$ as defined in [8]. The area inside $C$ and within $\Phi$ denotes the region $\sigma$, while the area outside $C$ and within $\Phi$ denotes the region $\Phi \backslash \sigma$.

$$
u_{N}(x, y)=\frac{u_{o}(x, y)-M_{1}}{M_{2}-M_{1}} \times M_{g}
$$

where $M_{1}$ and $M_{2}$ are the minima and maxima of $u_{o}$ among the neighborhoods. $M_{g}$ is the maximum gray level value of the original image. 
The local contrast will be more sensitive to noise with smaller neighborhood size. At a larger size, it will be less sensitive to noise and will lose fine detail.

The local term minimizes the variance of the local contrast inside and outside $C$, and we believe the original assumption that the object is statistically different from the background, including its local contrast. Due to uneven illumination, the global term alone will fail to capture the vessels. However, the local contrast between the vessels and the background remains unchanged. Hence, by applying the local term, the vessel can be completely extracted.

\section{Level Set Formulation}

In the level set formulation, the curve $C$ is represented by the zero level set of a Lipschitz function $\phi$. The overall energy function becomes $J(\phi)=\alpha F_{G}(\phi)+\beta F_{L}(\phi)$. We then use the Heaviside function $H$ and the Dirac Delta function $\delta_{o}$ as defined in [12] to partition the level set function. The overall energy function can be rewritten in terms of the $H$ in (4).

$$
\begin{aligned}
J(\phi)= & \alpha\left[\mu \int_{\Omega} \delta_{o}(\phi(x, y))|\nabla \phi(x, y)| d x d y\right. \\
& +\lambda_{1} \int_{\Omega}\left|u_{o}(x, y)-c_{1}\right|^{2} H(\phi(x, y)) d x d y \\
& \left.+\lambda_{2} \int_{\Omega}\left|u_{o}(x, y)-c_{2}\right|^{2}(1-H(\phi(x, y))) d x d y\right] \\
& +\beta\left[\lambda_{3} \int_{\Phi}\left|u_{N}(x, y)-m_{1}\right|^{2} H(\phi(x, y)) d x d y\right. \\
& \left.+\lambda_{4} \int_{\Phi}\left|u_{N}(x, y)-m_{2}\right|^{2}(1-H(\phi(x, y))) d x d y\right] .
\end{aligned}
$$

The averaging constant $c_{1}, c_{2}, m_{1}$, and $m_{2}$ can also be rewritten in terms of $H$ accordingly as in (5).

$$
\begin{aligned}
c_{1}(\phi) & =\frac{\int_{\Omega} u_{o}(x, y) H(\phi(x, y)) d x d y}{\int_{\Omega} H(\phi(x, y)) d x d y} \\
c_{2}(\phi) & =\frac{\int_{\Omega} u_{o}(x, y)(1-H(\phi(x, y))) d x d y}{\int_{\Omega}(1-H(\phi(x, y))) d x d y} \\
m_{1}(\phi) & =\frac{\int_{\Phi} u_{N}(x, y) H(\phi(x, y)) d x d y}{\int_{\Phi} H(\phi(x, y)) d x d y} \\
m_{2}(\phi) & =\frac{\int_{\Phi} u_{N}(x, y)(1-H(\phi(x, y))) d x d y}{\int_{\Phi}(1-H(\phi(x, y))) d x d y} .
\end{aligned}
$$

By keeping $c_{1}, c_{2}, m_{1}$, and $m_{2}$ fixed, and minimizing the overall energy function $J(\phi)$, the evolution equation can be expressed as in (6).

$$
\begin{aligned}
\frac{\partial \phi}{\partial t}=\delta_{o}(\phi)\{\alpha & {\left[\mu \operatorname{div}\left(\frac{\nabla \phi}{|\nabla \phi|}\right)\right.} \\
& \left.-\lambda_{1}\left(u_{o}(x, y)-c_{1}\right)^{2}+\lambda_{2}\left(u_{o}(x, y)-c_{2}\right)^{2}\right]_{(x, y) \in \Omega} \\
& +\beta\left[-\lambda_{3}\left(u_{N}(x, y)-m_{1}\right)^{2}\right. \\
& \left.\left.+\lambda_{4}\left(u_{N}(x, y)-m_{2}\right)^{2}\right]_{(x, y) \in \Phi}\right\} .
\end{aligned}
$$

We note that the local term with $\beta$ is only effective in the narrowband. In the numerical implementation, we put (6) in the form below,

$$
\left.\left.\begin{array}{l}
\frac{\phi_{i, j}^{n+1}-\phi_{i, j}^{n}}{\Delta t} \\
=\delta_{h}\left(\phi_{i, j}^{n}\right) \\
\quad \times\left\{\alpha \left[\frac{u}{h^{2}} \Delta_{-}^{x}\left(\frac{\Delta_{+}^{x} \phi_{i, j}^{n+1}}{\sqrt{\frac{\left(\Delta_{+}^{x} \phi_{i, j}^{n}\right)^{2}}{h^{2}}+\frac{\left(\phi_{i, j+1}^{n}-\phi_{i, j-1}^{n}\right)^{2}}{2 h^{2}}}}\right)\right.\right. \\
\quad+\frac{\mu}{h^{2}} \Delta_{-}^{y}\left(\frac{\Delta_{+}^{y} \phi_{i, j}^{n+1}}{\sqrt{\frac{\left(\Delta_{+}^{y} \phi_{i, j}^{n}\right)^{2}}{h^{2}}}+\frac{\left(\phi_{i+1, j}^{n}-\phi_{i-1, j}^{n}\right)^{2}}{2 h^{2}}}\right) \\
\left.\quad-\lambda_{1}\left(u_{o, i, j}-c_{1}\left(\phi^{n}\right)\right)^{2}+\lambda_{2}\left(u_{o, i, j}-c_{2}\left(\phi^{n}\right)\right)^{2}\right]
\end{array}\right]\right\}
$$

where $h$ is the space step and $\Delta t$ is the time step. In each iteration $n$, the narrowband $\Phi^{n}$ is updated according to the level set function $\phi^{n}$. We then fix $\Phi^{n}$ and $\phi^{n}$ for the calculations of $\Phi^{n+1}$, and $\phi^{n+1}$ numerically.

In the traditional level set method, the reinitialization of the level set function is required for each iteration. This prevents the level set function from being flattened. In our algorithm, we ignore the reinitialization process when applying the global term. This helps the level set function to develop and grow interior contours. However, when applying the local term in narrow band, the reinitialization process is essential to correctly define the narrow band.

\section{EXPERIMENTAL RESULTS}

In this section, two examples are shown to demonstrate the capability of the proposed model. They include a synthetic image to illustrate the idea and a clinical angiogram for feasibility study. The proposed model is compared with the Chan-Vese model. In the experiments, the parameters of the models are set as $\lambda_{1}=\lambda_{2}=\lambda_{3}=\lambda_{4}=1.0$, and the size of the neighborhood for local contrast enhancement is $9 \times 9$.

A synthetic image of a vessel branch with nonuniform illumination is shown in Fig. 1. The initial contour is set in the center of the image. The result of the Chan-Vese model shows that it cannot cope with the changes of the illuminations and fails to extract the vessel branch. This is because the model only measures the global statistics in the whole evolution process. When the intensity of the vessel closes to the mean intensity value of the background, the model treats those vessels as image background. In contrast, the proposed model adaptively improves the segmentation result across the nonuniform illumination areas and extracts the entire vessel branch correctly.

We further compare the models with a clinical angiogram as shown in Fig. 2. The initial contour and some labels are placed in Fig. 2(b). This is an $168 \times 168$-pixel angiogram of an aortic arch. The Chan-Vese model can only extract some portions of the right vertebral artery at location $\mathrm{A}$, the left carotid artery at location $\mathrm{B}$, and the left subclavian artery at location $\mathrm{C}$. At location $\mathrm{C}$, for example, it suffers from high nonuniformity in illumination. The proposed model, by contrast, is capable of extracting these vessels completely under nonuniform illumination. 


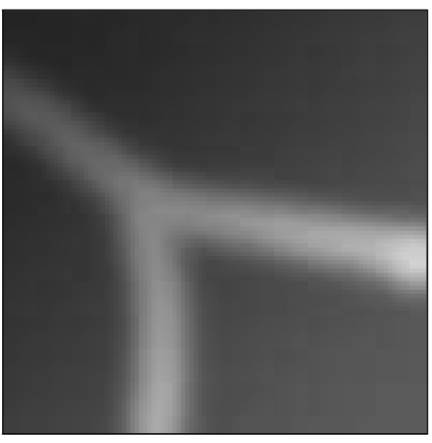

(a)

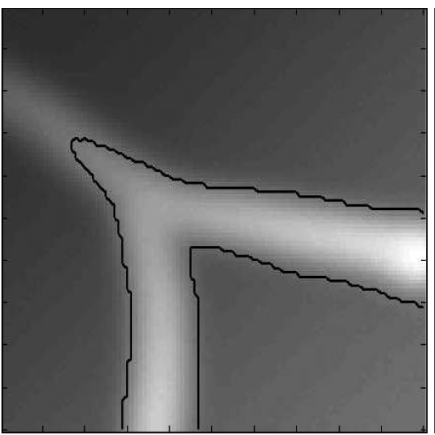

(c)

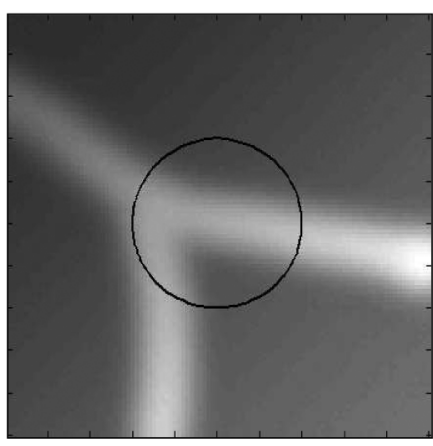

(b)

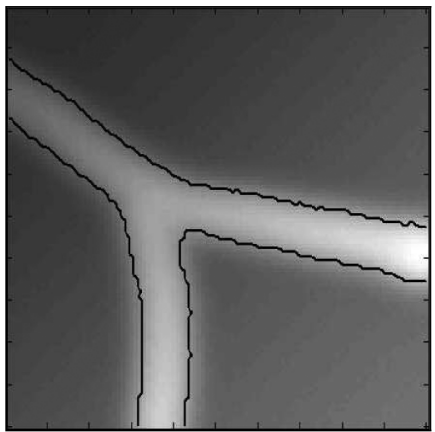

(d)
Fig. 1. (a) Synthetic image with nonuniform illumination. (b) Initial contour. (c) Result of the Chan-Vese model. (d) Result of the proposed model.

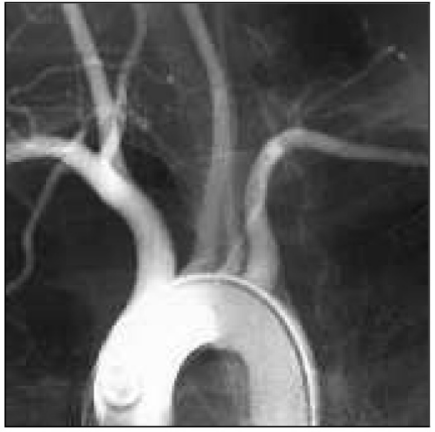

(a)

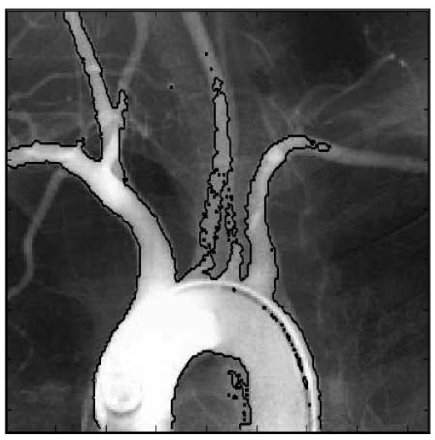

(c)

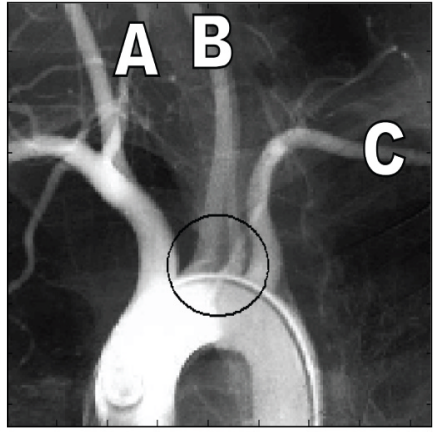

(b)

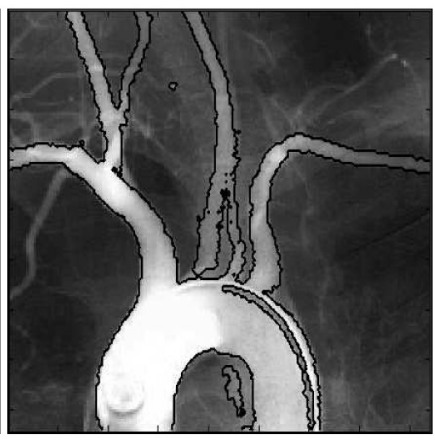

(d)
Fig. 2. (a) Aortic arch angiogram. (b) Initial contour and labels. (c) Result of the Chan-Vese model. (d) Result of the proposed model.

\section{CONCLUSION}

We have introduced a new active contour model for vessel extraction. This model is defined based on the techniques of curve evolutions by using the level set method. The new approach uses both regional statistics and local image contrast in the level set formulation. The global term helps to locate the vessel roughly, while the local term refines the detailed structures effectively. Applying the global term alone, as in the Chan-Vese model, fails to extract all the detailed vessels, but if the global term is ignored, the local term might oversegment the image. A combination of the two provides a better solution than using one or other term alone.

\section{REFERENCES}

[1] C. Kirbas and F. Quek, "A review of vessel extraction techniques and algorithms," ACM Comput. Surv., vol. 36, no. 2, pp. 81-121, Jun. 1, 2004.

[2] M. Kass, A. Witkin, and D. Terzopoulos, "Snakes: Active contour modes," Int. J. Comput. Vis., pp. 321-331, 1988.

[3] C. Xu and J. L. Prince, "Snakes, shapes, and gradient vector flow," IEEE Trans. Image Process., vol. 7, no. 3, pp. 359-369, Mar. 1998.

[4] K. W. Sum and P. Y. S. Cheung, "Boundary vector field for parametric active contours," Pattern Recogn., vol. 40, no. 6, pp. 1635-1645, 2007.

[5] A. Dervieux and F. Thomasset, "A finite element method for the simulation of Raleigh-Taylor instability," Springer Lecture Notes Math., vol. 771, pp. 145-158, 1980.

[6] S. Osher and J. A. Sethian, "Fronts propagating with curvature-dependent speed: Algorithms based on Hamilton-Jacobi formulation," $J$. Comput. Phys., vol. 79, pp. 12-49, 1988.

[7] K. W. Sum and P. Y. S. Cheung, "A novel active contour model using local and global statistics for vessel extraction," in 2006 Int. Conf. IEEE Eng. Medicine Biol. Soc. (EMBS), New York, Aug. 2006, pp. 3126-3129.

[8] R. Malladi, J. A. Sethian, and B. C. Vemuri, "Shape modeling with front propagation: A level set approach," IEEE Trans. Pattern Anal. Mach. Intell., vol. 17, no. 2, pp. 158-175, Feb. 1995.

[9] V. Caselles, F. Catte, T. Coll, and F. Dibos, "A geometric model for active contours in image processing," Numer. Math., vol. 66, no. 1, pp. 1-31, Dec. 1993.

[10] A. Yezzi, S. Kichenassamy, A. Kumar, P. Olver, and A. Tannenbaum, "A geometric snake model for segmentation of medical imagery," IEEE Trans. Med. Imag., vol. 16, no. 2, pp. 199-209, Apr. 1997.

[11] V. Caselles, R. Kimmel, and G. Sapiro, "On geodesic active contours," Int. J. Comput. Vis., vol. 22, no. 1, pp. 61-79, 1997.

[12] T. Chan and L. Vese, "Active contour without edges," IEEE Trans. Image Process., vol. 10, no. 2, pp. 266-277, Feb. 2001. 